\title{
STABILITY OF PATTERNS WITH ARBITRARY PERIOD FOR A GINZBURG-LANDAU EQUATION WITH A MEAN FIELD
}

\author{
JOHN NORBURY, JUNCHENG WEI, AND MATTHIAS WINTER
}

Abstract. We consider the following system of equations

$$
\left\{\begin{array}{l}
A_{t}=A_{x x}+A-A^{3}-A B, \quad x \in R, t>0, \\
B_{t}=\sigma B_{x x}+\mu\left(A^{2}\right)_{x x}, \quad x \in R, t>0
\end{array}\right.
$$

where $\mu>\sigma>0$. It plays an important role as a Ginzburg-Landau equation with a mean field in several fields of the applied sciences.

We study the existence and stability of periodic patterns with an arbitrary minimal period L. Our approach is by combining methods of nonlinear functional analysis such as nonlocal eigenvalue problems and the variational characterization of eigenvalues with Jacobi elliptic integrals. This enables us to give a complete characterization of existence and stability for all solutions with $A>0$, spatial average $<B>=0$ and an arbitrary minimal period.

\section{INTRODUCTION}

In this paper, we study the existence and stability of periodic solutions for the following amplitude equations

$$
\left\{\begin{array}{l}
A_{t}=A_{x x}+A-|A|^{2} A-A B, \quad x \in R, t>0, \\
B_{t}=\sigma B_{x x}+\mu\left(|A|^{2}\right)_{x x}, \quad x \in R, t>0
\end{array}\right.
$$

where

$$
\sigma>0, \quad \mu \in R, \quad x \in R,
$$

$A(x), B(x)$ have an arbitrary given minimal period $L>0, A(x)>0$ and

$$
<B>=\frac{1}{L} \int_{-L / 2}^{L / 2} B(x) d x=0 .
$$

Amplitude equations of the form (1.1) play a role in various areas of the applied sciences. In [3], the equation (1.1) was derived in the study of secondary stability of a one-dimensional cellular pattern. For a survey on hydrodynamics appliations see [5]. In [4] the system (1.1) was derived from various models arising in thermosolutal convection, rotating convection, or magnetoconvection.

It is important to understand (1.1) for finite $L$ since this makes a comparison possible between the amplitude equations and the full partial differential equation where they originate from. Although the amplitude equations are derived for the limit $L \rightarrow \infty$ they are expected to represent the behaviour of the full system also for finite $L$ and numerical simulations show that this is actually the case for many parameter regimes.

1991 Mathematics Subject Classification. Primary 35B35, 76E30; Secondary 35B40, 76E06.

Key words and phrases. Pattern Formation, Mean field, Stability, Steady-states. 
We now reduce system (1.1) to its final form. By taking $\tau=\frac{1}{\sigma}, \mu^{\prime}=\frac{\mu}{\sigma}$, the system (1.1) can be rewritten in the form

$$
\left\{\begin{array}{l}
A_{t}=A_{x x}+A-|A|^{2} A-A B, \quad x \in R, t>0, \\
\tau B_{t}=B_{x x}+\mu^{\prime}\left(|A|^{2}\right)_{x x}, \quad x \in R, t>0,
\end{array}\right.
$$

where

$$
\tau>0, \quad \mu^{\prime} \in R
$$

In the equations (1.2), $A$ may be complex. In this case one can decompose $A=R \exp (i \theta)$ with functions $R$ and $\theta$ representing the amplitude and the phase of $A$, respectively. An explicit analytical treatment of the general case is very complicated. In this paper we therefore restrict our attention to the invariant subspace in which $A$ is real.

We study periodic solutions of (1.2) with $A>0$. Assuming that the minimal period is some positive number $L>0$, we have

$$
A(x+L)=A(x), \quad B(x+L)=B(x) \quad \text { for all } x \in R
$$

and we have chosen the smallest such $L>0$.

Using Rolle's theorem, one sees that there exists an $x_{0} \in\left(-\frac{L}{2}, \frac{L}{2}\right)$ such that $A^{\prime}\left(x_{0}\right)=0$ and by periodicity we also have $A^{\prime}\left(x_{0}+L\right)=0$ and $A\left(x_{0}+L\right)=A\left(x_{0}\right)$. Further, by the structure of (1.2), after translation any solution with $A>0$ having minimal period $L$ can be represented by an even function on $\left(-\frac{L}{2}, \frac{L}{2}\right)$ with Neumann boundary conditions.

On the other hand, if we have a solution on an interval of length $\frac{L}{2}$ with Neumann boundary conditions, by even continuation we also get a periodic solution on the real line with period $L$.

We will use this representation of a periodic function with minimal period $L$ by a function on an interval of length $\frac{L}{2}$ with Neumann boundary conditions throughout the paper. Thus from now on we focus on getting even solutions on $\left(-\frac{L}{2}, \frac{L}{2}\right)$ with Neumann boundary conditions, or equivalently, solutions on $\left(0, \frac{L}{2}\right)$ with Neumann boundary conditions without imposing any symmetry.

Hence to get an understanding of all periodic solutions with arbitrary period $L$ it suffices to all study solutions on an interval of arbitrary length with Neumann boundary conditions.

In order to have nontrivial stable solutions we make the following assumption throughout the paper:

$$
\mu^{\prime}=\frac{\mu}{\sigma}>1
$$

We study these solutions by combining methods of nonlinear functional analysis such as nonlocal eigenvalue problems and the variational characterization of eigenvalues with Jacobi elliptic integrals.

Following this rigorous approach, we give a complete characterization of existence and stability for all periodic solutions with $A>0$.

We now state our main results on existence and stability of stationary patterns for system (1.2) with arbitrary minimal period $L>0$ as defined in (1.3). 
We first consider the existence of steady-state solutions with minimal period $L$. Namely, by the remarks above, we consider the following steady-state system

$$
\left\{\begin{array}{l}
A_{x x}+A-A^{3}-A B=0,0<x<\frac{L}{2}, \\
B_{x x}+\mu^{\prime}\left(A^{2}\right)_{x x}=0,0<x<\frac{L}{2} \\
A_{x}(0)=A_{x}\left(\frac{L}{2}\right)=0 \\
B_{x}(0)=B_{x}\left(\frac{L}{2}\right)=0,<B>=0 .
\end{array}\right.
$$

We have

Theorem 1. Let $L>0$ be a fixed number and $\mu^{\prime}>1$. Then there exist two numbers $\mu_{1}(L)>\mu_{2}(L)>1$ (to be given explicitly in (3.26) of Section 3) such that the following holds.

(1) If $\mu^{\prime}>\mu_{1}(L)$, all solutions of (1.5) with $A>0$ must be constant.

(2) If $\mu^{\prime}=\mu_{1}(L)$, there exists exactly one solution of (1.5) with $A>0$ which has minimal period $L$.

(3) If $\mu_{2}(L)<\mu^{\prime}<\mu_{1}(L)$, there exist exactly two solutions of (1.5) with $A>0$ which have minimal period $L$.

(4) If $1<\mu^{\prime} \leq \mu_{2}(L)$, there exists exactly one solution of (1.5) with $A>0$ which has minimal period $L$.

We now study stability. Our notion of stability is linearized stability among solutions with period $L$. The next theorem classifies the instability of large classes of steady-state solutions.

Theorem 2. All solutions of (1.5)

(1) which do not have minimal period $L$ or

(2) for which $A$ changes sign or

(3) for which $A_{x}$ changes sign

are linearly unstable.

Thus we need to study only the stability of those steady-state solutions with $A>0$ which have the minimal period $L$. They are all given by Theorem 1 .

We have

Theorem 3. Among the steady-state solutions of (1.2) with $A>0$ which have the minimal period $L$ (classified by Theorem 1), we have

(1) If $\mu^{\prime}=\mu_{1}(L)$, there exists exactly one solution with $A>0$ having minimal period L. This solution is neutrally stable.

(2) If $\mu_{2}(L)<\mu^{\prime}<\mu_{1}(L)$, there exist exactly two solutions with $A>0$ having minimal period $L$. The one with small amplitude $A(0)$ is linearly stable and the one with large amplitude $A(0)$ is linearly unstable.

(3) If $1<\mu^{\prime} \leq \mu_{2}(L)$, there exists exactly one solution with $A>0$ having minimal period $L$ and this solution is linearly unstable.

The explicit values of $\mu_{1}(L), \mu_{2}(L)$ are given in Section 3. In particular,

$$
\mu_{2}(L)=1+\frac{2 \pi^{2}}{L^{2}}
$$


and $\mu_{1}(L)$ is given in terms of elliptic integrals. Both $\mu_{1}(L)$ and $\mu_{2}(L)$ are functions of $L$ - the domain size - only. Thus we have rigorously established the following bifurcation picture (see also Figure 3 of $[7])$.

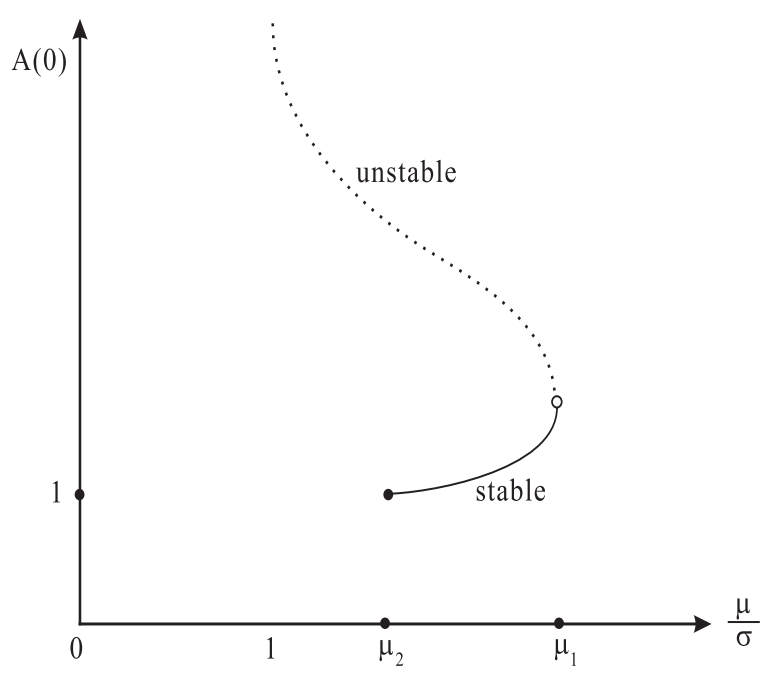

Figure 1. Bifurcation Curve

Theorem 1 completely classifies the existence of steady-state solutions with $A>0$ having minimal period $L$. Moreover, the stability or instability of all steady-state solutions is given in Theorem 2 and Theorem 3.

Recall that we do not have any condition on the minimal period $L$. Our results show that nontrival stable patterns exist for a Ginzburg-Landau equation coupled to an equation for a mean field, even when the coefficients of the equations are real and when the minimal period is finite.

Previous numerical and analytical studies of these amplitude equation include [7] (numerical simulation, asymptotic expansion and bifurcation theory, in particular the use of Jacobi elliptic integrals to describe the shape of solutions and a numerical study of stability) and [8] (rigorous study of the limit when the minimal period is large enough). In [8] the resulting steady-states are pulses or spikes and nonlocal eigenvalue problems are used, but no Jacobi elliptic integrals. In particular, we showed in [8] that there are two large single pulse solutions, where one is stable and the other one is unstable.

Another type of Ginzburg-Landau equation, where the term $\left(|A|^{2}\right)_{x x}$ in the $B$-equation is replaced by $\partial_{x}\left(|A|^{2}\right)$ has been considered by several authors, see [9] and the references therein. In that case the basic patterns are travelling pulses which arise in the convection of binary fluids.

The organization of this paper is as follows:

In Section 2, we perform a scaling argument and reduce the existence problem to an algebraic equation for consistency.

In Section 3, we use elliptic integrals to rigorously solve the algebraic equation for consistency and prove Theorem 1. 
In Section 4, the spectrum of a linearized operator is analyzed and a key identity is derived.

In Section 5, we study a crucial nonlocal eigenvalue problem.

Section 6 contains the proof of Theorem 3, the main result on stability.

In Section 7, we prove Theorem 2 by showing the instability of all other solutions, invoking the variational characterization of eigenvalues.

In the Appendix, the linear operator is first derived and then reduced to a self-adjoint nonlocal eigenvalue problem which is given in the reduction lemma, Lemma 13.

Acknowledgments. The research of JW is supported by an Earmarked Grant from RGC of Hong Kong. JN and MW thank the Department of Mathematics at The Chinese University of Hong Kong for their kind hospitality.

\section{ScAling AND CONSISTENCy ALGEBRAiC EQUATION}

In this section, we rescale the steady-state equation (1.5) and reduce it to a single ordinary differential equation coupled with an algebraic equation for consistency.

Consider the steady states of the equations (1.2). From (1.5) we derive

$$
B(x)=-\mu^{\prime} A^{2}(x)+\mu^{\prime}<A^{2}>, \quad<A^{2}>=\frac{1}{L} \int_{I} A^{2}(x) d x
$$

where $I=\left(-\frac{L}{2}, \frac{L}{2}\right)$. Substituting (2.1) into the first equation of (1.5), we obtain

$$
\left\{\begin{array}{l}
A_{x x}-a A+b A^{3}=0, \quad-\frac{L}{2}<x<\frac{L}{2}, \\
A(x) \text { has minimal period } L,
\end{array}\right.
$$

where

$$
a=\mu^{\prime}<A^{2}>-1, \quad b=\mu^{\prime}-1
$$

We consider $a$ as a real parameter. Since (2.2) is an autonomous equation, as was explained in the introduction, we may assume that $A$ satisfies the following boundary, symmetry, and positivity conditions:

$$
\begin{gathered}
A^{\prime}\left(-\frac{L}{2}\right)=A^{\prime}\left(\frac{L}{2}\right)=0, \quad A(x)=A(-x), \\
A^{\prime}(x)<0 \text { for } 0<x<\frac{L}{2} .
\end{gathered}
$$

We now solve (2.2) with consistency condition (2.3). Note that a positive solution of (2.2) exists if and only if $a>0$ (since $b>0)$. In that case, we let $\beta=\sqrt{a}$ and

$$
A(x)=\frac{\beta}{\sqrt{\mu^{\prime}-1}} w_{l}(y),
$$

where

$$
y=\beta x, \quad \frac{\beta L}{2}=l,
$$

and $w_{l}$ solves the following boundary value problem:

$$
w_{l}^{\prime \prime}-w_{l}+w_{l}^{3}=0, \quad w_{l}^{\prime}(0)=w_{l}^{\prime}(l)=0,
$$




$$
\begin{aligned}
& w_{l}^{\prime}(y)<0 \text { for } 0<y<l, \\
& w_{l}(y)>0 \quad \text { for } 0<y<l .
\end{aligned}
$$

In Lemma 5 we will show that (2.7) has a unique solution if $l>\pi / \sqrt{2}$ and no solution if $l \leq \pi / \sqrt{2}$.

Substituting (2.5) into (2.3), it is easy to see that $\beta$ will have to satisfy the following consistency equation:

$$
\beta^{2}-\frac{2 \mu^{\prime}}{L\left(\mu^{\prime}-1\right)} \beta \int_{0}^{\frac{\beta L}{2}} w_{l}^{2} d y+1=0 .
$$

We write $(2.8)$ in terms of the new length $l=\frac{\beta L}{2}$ :

$$
l^{2}-t l \int_{0}^{l} w_{l}^{2} d y+\frac{L^{2}}{4}=0, \text { where } t=\frac{\mu^{\prime}}{\mu^{\prime}-1} .
$$

In (2.9), $t$ is a fixed parameter, $L$ is a given parameter (which corresponds to the domain size), and $l$ is the unknown. Note that the unknown $l$ appears in the equation (2.9) in three ways: explicitly, as the upper boundary of the integral and as the index of the function $w_{l}$. The last two dependencies are new compared to the singular limit case $L>>1$ and they make this study more difficult than the singular limit case.

If there exists a solution $l$ to $(2.9)$, then, by taking

$$
A(x)=\frac{2 l}{L \sqrt{\mu^{\prime}-1}} w_{l}\left(\frac{2 l x}{L}\right),
$$

we obtain a solution of (2.2). Conversely, by taking (2.10), $l$ must satisfy (2.9). Thus we have reduced our problem to showing existence of (2.7) (see Lemma 5) and solving the algebraic equation (2.9) for consistency.

\section{Solving the CONSISTEnCy EQUATION}

In this section, we show that (2.7) has a unique solution (see Lemma 5) and solve the consistency equation (2.9). Our idea is to represent the ordinary differential equation (2.7) by Jacobi elliptic integrals. Then we use their properties to show that (2.7) has a unique solution and to solve the algebraic equation (2.9).

Let $w_{l}(0)=M$ and $w_{l}(l)=m$. Recall that $0<m<M$. From (2.7), we have

$$
\begin{gathered}
\left(w_{l}^{\prime}\right)^{2}=w_{l}^{2}-\frac{1}{2} w_{l}^{4}-M^{2}+\frac{1}{2} M^{4}=\frac{1}{2}\left(w_{l}^{2}-m^{2}\right)\left(M^{2}-w_{l}^{2}\right), \\
-m^{2}+\frac{1}{2} m^{4}=-M^{2}+\frac{1}{2} M^{4} .
\end{gathered}
$$

From (3.2), we deduce that

$$
M^{2}+m^{2}=2
$$

Note that

$$
l=\int_{m}^{M} \frac{d w_{l}}{\sqrt{\frac{1}{2}\left(w_{l}^{2}-m^{2}\right)\left(M^{2}-w_{l}^{2}\right)}}
$$


and

$$
\int_{0}^{l} w_{l}^{2} d y=\int_{m}^{M} \frac{w_{l}^{2} d w_{l}}{\sqrt{\frac{1}{2}\left(w_{l}^{2}-m^{2}\right)\left(M^{2}-w_{l}^{2}\right)}} .
$$

We now represent the values of the function $w_{l}$ by a phase function $\varphi$ such that the relations (3.4), (3.5) can be expressed by Jacobi elliptic integrals.

Let

$$
\frac{M^{2}+m^{2}}{2}-w_{l}^{2}=-\frac{M^{2}-m^{2}}{2} \cos (2 \varphi)
$$

Then it is easy to see that (3.4) and (3.5) become

$$
l=\sqrt{2-k^{2}} K(k), \quad \int_{0}^{l} w_{l}^{2} d y=\frac{2}{\sqrt{2-k^{2}}} E(k),
$$

where

$$
\frac{1}{M}=\sqrt{1-\frac{k^{2}}{2}}
$$

and $E(k)$ and $K(k)$ are Jacobi elliptic integrals:

$$
\begin{aligned}
& E(k)=\int_{0}^{\frac{\pi}{2}} \sqrt{1-k^{2} \sin ^{2} \varphi} d \varphi, \\
& K(k)=\int_{0}^{\frac{\pi}{2}} \frac{1}{\sqrt{1-k^{2} \sin ^{2} \varphi}} d \varphi .
\end{aligned}
$$

For the properties of elliptic integrals, we refer the reader to [1] and [2]. We list the following for later use:

$$
\begin{gathered}
E(0)=\frac{\pi}{2}, \quad K(0)=\frac{\pi}{2}, \quad \lim _{k \rightarrow 1} E(k)=1, \quad \lim _{k \rightarrow 1} K(k)=+\infty, \\
k^{\prime} K(k)<E(k)<\left(1-\frac{k^{2}}{2}\right) K(k)<K(k), \\
\frac{d K(k)}{d k}=\frac{E(k)-\left(k^{\prime}\right)^{2} K(k)}{k\left(k^{\prime}\right)^{2}}, \quad \frac{d E(k)}{d k}=\frac{E(k)-K(k)}{k},
\end{gathered}
$$

where

$$
0<k<1 \text { and } k^{\prime}=\sqrt{1-k^{2}} .
$$

By (3.6), our consistency equation (2.9) can be rewritten in terms of the new variable $k \in(0,1)$ :

$$
\left(1-\frac{k^{2}}{2}\right) K^{2}(k)-t E(k) K(k)+\frac{L^{2}}{8}=0 .
$$

Thus the existence problem is reduced to solving (3.11) for $k$.

We begin with

Lemma 4. For $0<k<1$, we have

$$
\begin{gathered}
\frac{d k}{d l}>0 \\
\frac{d}{d k}\left[\left(1-\frac{k^{2}}{2}\right) K^{2}(k)\right]>0,
\end{gathered}
$$




$$
\begin{gathered}
\frac{d^{2}}{d k^{2}}\left[\left(1-\frac{k^{2}}{2}\right) K^{2}(k)\right]>0, \\
\frac{d}{d k}[E(k) K(k)]>0, \\
\frac{d^{2}}{d k^{2}}[E(k) K(k)]<0, \\
\frac{d^{2}}{d k^{2}}\left[\frac{\left(1-\frac{k^{2}}{2}\right) K(k)}{E(k)}\right]>0, \\
\frac{d^{2}}{d k^{2}}\left[\frac{1}{E(k) K(k)}\right]>0 .
\end{gathered}
$$

Proof: The proof is mainly based on the inequality (3.9).

Using (3.10), we arrive at

$$
\frac{d}{d k}\left[\left(1-\frac{k^{2}}{2}\right) K^{2}\right]=\frac{K}{k\left(k^{\prime}\right)^{2}}\left[\left(1+\left(k^{\prime}\right)^{2}\right) E-2\left(k^{\prime}\right)^{2} K\right]>0
$$

by (3.9). Thus (3.13) is proved. Since $l^{2}=\left(2-k^{2}\right) K^{2}$, (3.12) follows from (3.13).

Equation (3.14) is more difficult to prove. In fact, by lengthy computations, we have

$$
\begin{gathered}
\frac{d^{2}}{d k^{2}}\left[\left(1-\frac{k^{2}}{2}\right) K^{2}\right] \\
=\frac{1}{k^{2}\left(k^{\prime}\right)^{4}}\left[\left(1+\left(k^{\prime}\right)^{2}\right) E^{2}+E K\left(2-7\left(k^{\prime}\right)^{2}-\left(k^{\prime}\right)^{4}\right)+\left(k^{\prime}\right)^{2} K^{2}\left(5\left(k^{\prime}\right)^{2}-1\right)\right] .
\end{gathered}
$$

It is easy to see that if $5\left(k^{\prime}\right)^{2}-1 \leq 0$, then

$$
\begin{gathered}
\left(1+\left(k^{\prime}\right)^{2}\right) E^{2}+E K \\
\left(2-7\left(k^{\prime}\right)^{2}-\left(k^{\prime}\right)^{4}\right)+\left(k^{\prime}\right)^{2} K^{2}\left(5\left(k^{\prime}\right)^{2}-1\right) \\
>E^{2}-\left(k^{\prime}\right)^{2} K^{2}>0 .
\end{gathered}
$$

Now we assume that $5\left(k^{\prime}\right)^{2}-1>0$. Then, using (3.9), we have

$$
\begin{aligned}
& \left(1+\left(k^{\prime}\right)^{2}\right) E^{2}+E K\left(2-7\left(k^{\prime}\right)^{2}-\left(k^{\prime}\right)^{4}\right)+\left(k^{\prime}\right)^{2} K^{2}\left(5\left(k^{\prime}\right)^{2}-1\right) \\
= & \left(1+\left(k^{\prime}\right)^{2}\right) E\left(E-k^{\prime} K\right)+\left(k^{\prime}\right)^{2}\left(5\left(k^{\prime}\right)^{2}-1\right) K\left(K-\frac{E}{1-\frac{k^{2}}{2}}\right) \\
+ & E K\left[k^{\prime}\left(1+\left(k^{\prime}\right)^{2}\right)+2-7\left(k^{\prime}\right)^{2}-\left(k^{\prime}\right)^{4}+\frac{1}{1-\frac{k^{2}}{2}}\left(k^{\prime}\right)^{2}\left(5\left(k^{\prime}\right)^{2}-1\right)\right] .
\end{aligned}
$$

For the last term in (3.20), we have

$$
\begin{gathered}
k^{\prime}\left(1+\left(k^{\prime}\right)^{2}\right)+2-7\left(k^{\prime}\right)^{2}-\left(k^{\prime}\right)^{4}+\frac{1}{1-\frac{k^{2}}{2}}\left(k^{\prime}\right)^{2}\left(5\left(k^{\prime}\right)^{2}-1\right) \\
>k^{2}\left(2-5\left(k^{\prime}\right)^{2}\right)+\left(k^{\prime}-\left(k^{\prime}\right)^{2}\right)\left(1+\left(k^{\prime}\right)^{2}\right)+\frac{k^{2}}{2}\left(k^{\prime}\right)^{2}\left(5\left(k^{\prime}\right)^{2}-1\right)>\frac{5}{2} k^{4}>0 .
\end{gathered}
$$

Thus (3.14) is proved. 
By using (3.10), it is easy to see that

$$
\frac{d}{d k}[E K]=\frac{E^{2}-\left(k^{\prime}\right)^{2} K^{2}}{k\left(k^{\prime}\right)^{2}}
$$

which is positive by (3.9). This proves (3.15).

We calculate

$$
\frac{d^{2}}{d k^{2}}(E K)=\frac{1}{k^{2}\left(k^{\prime}\right)^{2}}\left[2(E-K)^{2}-\left(E^{2}-\left(k^{\prime}\right)^{2} K^{2}\right) \frac{1-\frac{2}{3} k^{2}}{\left(k^{\prime}\right)^{2}}\right] .
$$

Note that

$$
\begin{aligned}
& \left(E^{2}-\left(k^{\prime}\right)^{2} K^{2}\right)\left(1-\frac{2}{3} k^{2}\right)-2\left(k^{\prime}\right)^{2}(E-K)^{2} \\
> & \left(k^{\prime}\right)^{2}\left(E^{2}-K^{2}\right)\left(1-\frac{2}{3} k^{2}\right)-2\left(k^{\prime}\right)^{2}(E-K)^{2} \\
= & \left(k^{\prime}\right)^{2}(E-K)\left[(E+K)\left(1-\frac{2}{3} k^{2}\right)-2(E-K)\right] \\
= & \left(k^{\prime}\right)^{2}(E-K)\left[K\left(3-\frac{2}{3} k^{2}\right)-\left(1+\frac{2}{3} k^{2}\right) E\right]>0
\end{aligned}
$$

by (3.9), which proves (3.16).

Finally, (3.17) and (3.18) follow from (3.13) - (3.16) by simple calculus.

Lemma 5. If $l>\pi / \sqrt{2}$ the equation (2.7) has a unique solution. If $l \leq \pi / \sqrt{2}$ the equation (2.7) has no solution.

Proof: The inequality (3.12) implies that to every $k$ with $0<k<1$ there belongs exactly one $l>0$. By definition $0<k<1$ parameterizes all solutions of (2.7) with $1<M<\sqrt{2}$. Now (3.6) and (3.8) imply that the solutions are also parameterized by $\pi / \sqrt{2}<l<+\infty$. For any $l \leq \pi / \sqrt{2}$ the function $w_{l}=1$ satisfies all requirements of $(2.7)$ except $w^{\prime}(y)<0$ for $0<y<l$ and therefore it is not a solution.

\section{Remarks:}

1.) Note that in Lemma 5 the functions with $w(0)=M, M>\sqrt{2}$ do not solve (2.7) since they do not satisfy $w(y)>0$ for all $0<y<l$. By Theorem 2, this would lead to an unstable solution of (1.5). Therefore we do not study this type of solution any further.

2.) The solution with $w(0)=\sqrt{2}$ corresponds to $l=+\infty$ and it does not solve (2.7) either, since there is no $0<l<+\infty$ with $w^{\prime}(l)=0$. Therefore this leads to a homoclinic connection which is defined on the whole line.

Now we rewrite (3.11) as follows:

$$
t=f(k) \equiv \frac{L^{2}}{8 E(k) K(k)}+\frac{\left(1-\frac{k^{2}}{2}\right) K(k)}{E(k)},
$$

where $f(k)$ is defined on $(0,1)$. By Lemma 4 and (3.8), we have

$$
\frac{d^{2}}{d k^{2}} f(k)>0
$$


and

$$
\begin{gathered}
f(0)=\frac{L^{2}}{8 E(0) K(0)}+\frac{K(0)}{E(0)}=\frac{L^{2}}{2 \pi^{2}}+1, \\
\lim _{k \rightarrow 1, k<1} f(k)=+\infty .
\end{gathered}
$$

By $(3.22),(3.23)$ and $(3.24)$, the function $f(k)$ is strictly convex and attains a unique minimum at a point $k_{0} \in(0,1)$ such that

$$
f^{\prime}\left(k_{0}\right)=\left.\frac{L^{2}}{8} \frac{d}{d k}\left(\frac{1}{2 E(k) K(k)}\right)\right|_{k=k_{0}}+\left.\frac{d}{d k}\left(\frac{\left(1-\frac{k^{2}}{2}\right) K}{E}\right)\right|_{k=k_{0}}=0 .
$$

Furthermore, by strict convexity, for $k<k_{0}, f^{\prime}(k)<0$ and for $k>k_{0}, f^{\prime}(k)>0$.

Let us denote

$$
t_{1}(L)=\min _{k \in(0,1)} f(k), \quad t_{2}(L)=f(0)=\frac{L^{2}}{2 \pi^{2}}+1 .
$$

Correspondingly, we define

$$
\mu_{1}(L)=\frac{t_{1}}{t_{1}-1}, \quad \mu_{2}(L)=\frac{t_{2}}{t_{2}-1}=1+\frac{2 \pi^{2}}{L^{2}} .
$$

Summarizing these results, we obtain

Lemma 6. Let $L$ be fixed and $t_{1}<t_{2}$ be given in (3.25). Then we have

(a) Problem (3.11) has a solution if and only if $t \geq t_{1}$.

(b) For $t=t_{1}$, problem (3.11) has a unique solution $k_{0}$ and we have $f^{\prime}\left(k_{0}\right)=0$.

(c) For $t_{1}<t<t_{2}$, problem (3.11) has two solutions $k_{1}<k_{2}$. Moreover, we have

$$
f^{\prime}\left(k_{1}\right)<0, \quad f^{\prime}\left(k_{2}\right)>0 .
$$

(d) For $t \geq t_{2}$, problem (3.11) has a unique solution $k_{0}$ and we have $f^{\prime}\left(k_{0}\right)>0$.

Going back to (2.9), we express the results of Lemma 6 in terms of $L$ and $\mu^{\prime}$.

Lemma 7. Let $L$ be fixed and $\mu_{1}>\mu_{2}$ be given in (3.26). Then we have

(a) Problem (2.9) has a solution if and only if $1<\mu^{\prime} \leq \mu_{1}$.

(b) For $\mu^{\prime}=\mu_{1}$, problem (2.9) has a unique solution $l_{0}$ and we have $\frac{d \tilde{f}}{d l}\left(l_{0}\right)=0$.

(c) For $\mu_{2}<\mu^{\prime}<\mu_{1}$, problem (2.9) has two solutions $l_{1}<l_{2}$. Moreover, we have

$$
\frac{d \tilde{f}}{d l}\left(l_{1}\right)<0, \quad \frac{d \tilde{f}}{d l}\left(l_{2}\right)>0,
$$

where

$$
\tilde{f}(l)=l^{2}-t l \int_{0}^{l} w_{l}^{2} d y+\frac{L^{2}}{4} .
$$

(d) For $1<\mu^{\prime} \leq \mu_{2}$, problem (2.9) has a unique solution $l_{0}$ and we have $\frac{d \tilde{f}}{d l}\left(l_{0}\right)>0$.

Theorem 1 now follows from Lemma 7.

Thus we have rigorously derived a complete picture of the existence of periodic solutions with $A>0$. 


\section{Spectral ANALYSis AND A KEY IDENTITY}

Let $w_{l}$ be the unique solution of (2.7). We define the linear operator:

$$
\mathcal{L}[\phi]=\phi^{\prime \prime}-\phi+3 w_{l}^{2} \phi, \phi \in \mathcal{X}_{l},
$$

where

$$
\mathcal{X}_{l}=\left\{\phi \in H^{1}(-l, l) \mid \phi(-x)=\phi(x), \phi^{\prime}(-l)=\phi^{\prime}(l)=0\right\},
$$

or equivalently by a slight double use of notation

$$
\mathcal{X}_{l}=\left\{\phi \in H^{1}(0, l) \mid \quad \phi^{\prime}(0)=\phi^{\prime}(l)=0\right\}
$$

In this section, we analyze the spectrum of $\mathcal{L}$. The following lemma will be useful in the study of the stability of solutions of (1.5).

Lemma 8. Consider the following eigenvalue problem:

$$
\left\{\begin{array}{l}
\mathcal{L} \phi=\lambda \phi, \quad 0<y<l \\
\phi^{\prime}(0)=\phi^{\prime}(l)=0
\end{array}\right.
$$

Then $\lambda$ can be arranged in such a way that

$$
\lambda_{1}>0, \quad \lambda_{j}<0, \quad j=2, \ldots .
$$

Moreover, the eigenfunction corresponding to $\lambda_{1}$ (denoted by $\Phi_{1}$ ) can be made positive.

Proof: Let the eigenvalues of $\mathcal{L}$ be arranged by $\lambda_{1} \geq \lambda_{2} \geq \ldots$. It is well-known that $\lambda_{1}>\lambda_{2}$ and that the eigenfunction corresponding to $\lambda_{1}$ can be made positive. Moreover,

$$
\begin{gathered}
-\lambda_{1}=\min _{\int_{0}^{l} \phi^{2} d y=1}\left(\int_{0}^{l}\left[\left|\phi^{\prime}\right|^{2}+\phi^{2}-3 w_{l}^{2} \phi^{2}\right] d y\right) \\
\leq\left(\int_{0}^{l} w_{l}^{2} d y\right)^{-1}\left(\int_{0}^{l}\left[\left|w_{l}^{\prime}\right|^{2}+w_{l}^{2}-3 w_{l}^{2} w_{l}^{2}\right] d y\right)<0 .
\end{gathered}
$$

Next we claim that $\lambda_{2} \leq 0$. This follows from a classical argument (see Theorem 2.11 of [6]). For the sake of completeness, we include a proof here. By the variational characterization of $\lambda_{2}$, we have

$$
\begin{gathered}
-\lambda_{2}= \\
=\sup _{v \in H^{1}(I)} \inf _{\phi \in H^{1}(I), \phi \neq 0}\left[\frac{\int_{0}^{l}\left(\left(\phi^{\prime}\right)^{2}+\phi^{2}-3 w_{l}^{2} \phi^{2}\right) d y}{\int_{0}^{l} \phi^{2} d y} \mid v \not \equiv 0, \int_{0}^{1} \phi v d y=0\right] .
\end{gathered}
$$

On the other hand, $w_{l}$ has least energy, that is

$$
E\left[w_{l}\right]=\inf _{u \neq 0, u \in H^{1}(I)} E[u],
$$

where

$$
E[u]=\frac{\int_{0}^{l}\left(\left(u^{\prime}\right)^{2}+u^{2}\right) d y}{\left(\int_{0}^{l} u^{4} d y\right)^{\frac{1}{2}}} .
$$

Let

$$
h(t)=E\left[w_{l}+t \phi\right], \quad \phi \in H^{1}(0, l) .
$$


Then $h(t)$ attains its minimum at $t=0$ and hence

$$
\begin{aligned}
h^{\prime \prime}(0)=2\left[\int _ { 0 } ^ { l } \left(\left|\phi^{\prime}\right|^{2}\right.\right. & \left.\left.+\phi^{2}\right) d y-3 \int_{0}^{l} w_{l}^{2} \phi^{2} d y+2 \frac{\left(\int_{0}^{l} w_{l}^{3} \phi d y\right)^{2}}{\int_{0}^{l} w_{l}^{4} d y}\right] \\
& \times \frac{1}{\left(\int_{0}^{l} w_{l}^{4} d y\right)^{1 / 2}} \geq 0 .
\end{aligned}
$$

By (4.5), we see that

$$
\begin{gathered}
-\lambda_{2} \geq \inf _{\int_{0}^{l} \phi w_{l}^{3}=0, \phi \neq 00}\left[\int_{0}^{l}\left(\left|\phi^{\prime}\right|^{2}+\phi^{2}\right) d y-3 \int_{0}^{l} w_{l}^{2} \phi^{2} d y+2 \frac{\left(\int_{0}^{l} w_{l}^{3} \phi d y\right)^{2}}{\int_{0}^{l} w_{l}^{4} d y}\right] \\
\times \frac{1}{\int_{0}^{l} \phi^{2} d y} \geq 0 .
\end{gathered}
$$

Finally we claim that $\lambda_{2}<0$. But this follows from the proof of uniqueness of $w_{l}$, see Lemma 5 .

By Lemma $8, \mathcal{L}^{-1}$ exists and hence $\mathcal{L}^{-1} w_{l}$ is well-defined. Our next goal in this section is to compute the integral $\int_{0}^{l} w_{l} \mathcal{L}^{-1} w_{l} d y$ and thus to derive the following key identity.

Lemma 9. We have

$$
\int_{0}^{l} w_{l}\left(\mathcal{L}^{-1} w_{l}\right) d y=\frac{1}{4} \int_{0}^{l} w_{l}^{2} d y+\frac{1}{4} l \frac{d}{d l} \int_{0}^{l} w_{l}^{2} d y=\frac{1}{4} \frac{d}{d l}\left(l \int_{0}^{l} w_{l}^{2} d y\right) .
$$

\section{Proof:}

Let us denote $\phi_{l}=\mathcal{L}^{-1} w_{l}$. Then $\phi_{l}$ satisfies

$$
\phi_{l}^{\prime \prime}-\phi_{l}+3 w_{l}^{2} \phi_{l}=w_{l}, \quad \phi_{l}^{\prime}(0)=\phi_{l}^{\prime}(l)=0 .
$$

Set

$$
\phi_{l}=\frac{1}{2} w_{l}+\frac{1}{2} y w_{l}^{\prime}(y)+\Psi
$$

Then $\Psi(y)$ satisfies

$$
\begin{gathered}
\Psi^{\prime \prime}-\Psi+3 w_{l}^{2} \Psi=0, \\
\Psi^{\prime}(0)=0, \quad \Psi^{\prime}(l)=-\frac{1}{2} l w_{l}^{\prime \prime}(l) .
\end{gathered}
$$

On the other hand, let $\Psi_{0}=\frac{\partial w_{l}}{\partial M}$. Then $\Psi_{0}$ satisfies

$$
\begin{aligned}
& \Psi_{0}^{\prime \prime}-\Psi_{0}+3 w_{l}^{2} \Psi_{0}=0 \\
& \Psi_{0}(0)=1, \quad \Psi_{0}^{\prime}(0)=0 .
\end{aligned}
$$

Integrating (4.10), we have

$$
\begin{gathered}
\Psi_{0}^{\prime}(l)=\int_{0}^{l} \frac{\partial w_{l}}{\partial M} d y-3 \int_{0}^{l} w_{l}^{2} \frac{\partial w_{l}}{\partial M} d y \\
=\frac{d}{d M}\left(\int_{0}^{l}\left(w_{l}-w_{l}^{3}\right) d y\right)-\left(w_{l}(l)-w_{l}^{3}(l)\right) \frac{d l}{d M}
\end{gathered}
$$


Using the equation for $w_{l}$, we have $\int_{0}^{l}\left(w_{l}-w_{l}^{3}\right) d y=0$. Thus we obtain

$$
\Psi_{0}^{\prime}(l)=-\left(w_{l}(l)-w_{l}^{3}(l)\right) \frac{d l}{d M} .
$$

Comparing (4.9) and (4.11), we derive the following important relation:

$$
\Psi(x)=\frac{1}{2} \frac{l}{\frac{d l}{d M}} \Psi_{0}(x) .
$$

Hence, we have

$$
\begin{gathered}
\int_{0}^{l} w_{l} \phi_{l} d y=\int_{0}^{l}\left(\frac{1}{2} w_{l}+\frac{1}{2} y w_{l}^{\prime}+\Psi\right) w_{l} d y \\
=\frac{1}{4} \int_{0}^{l} w_{l}^{2} d y+\frac{1}{4} l w_{l}^{2}(l)+\frac{l}{2}\left(\frac{d l}{d M}\right)^{-1} \int_{0}^{l} w_{l} \Psi_{0} d y .
\end{gathered}
$$

On the other hand,

$$
\begin{aligned}
& \int_{0}^{l} w_{l} \Psi_{0} d y=\int_{0}^{l} w_{l} \frac{\partial w_{l}}{\partial M} d y \\
= & \frac{1}{2} \frac{d}{d M} \int_{0}^{l} w_{l}^{2} d y-\frac{1}{2} w_{l}^{2}(l) \frac{d l}{d M} \\
= & \frac{1}{2}\left[\frac{d}{d l} \int_{0}^{l} w_{l}^{2} d y-\frac{1}{2} w_{l}^{2}(l)\right] \frac{d l}{d M} .
\end{aligned}
$$

Substituting (4.14) into (4.13), we obtain that

$$
\begin{aligned}
\int_{0}^{l} w_{l} \phi_{l} d y & =\frac{1}{4} \int_{0}^{l} w_{l}^{2} d y+\frac{1}{4} l \frac{d}{d l} \int_{0}^{l} w_{l}^{2} d y \\
& =\frac{1}{4} \frac{d}{d l}\left(l \int_{0}^{l} w_{l}^{2} d y\right) .
\end{aligned}
$$

This finishes the proof of the lemma.

\section{A NONLOCAL EigenVAlue PROBlem}

Let $(A, B)$ be the solution of (1.2) with minimal period $L$. By Lemma 13 given in the appendix, to study the stability or instability of $(A, B)$, we just need to consider the following nonlocal eigenvalue problem

$$
\left\{\begin{array}{l}
\phi_{x x}-a \phi+3 b A^{2} \phi-2 \mu^{\prime}<A \phi>A=\lambda \phi, \quad-\frac{L}{2}<x<\frac{L}{2}, \\
\phi \in \mathcal{X}_{L},
\end{array}\right.
$$

where

$$
\mathcal{X}_{L}=\left\{\phi \in H^{1}\left(-\frac{L}{2}, \frac{L}{2}\right) \mid \phi(-x)=\phi(x), \phi^{\prime}\left(-\frac{L}{2}\right)=\phi^{\prime}\left(\frac{L}{2}\right)=0\right\} .
$$

Since $\phi(-x)=\phi(x)$, we may assume that $\phi^{\prime}(0)=0$ and solve $(5.1)$ for $0<x<\frac{L}{2}$.

Recall the following relation,

$$
A(x)=\frac{l}{L\left(\sqrt{\mu^{\prime}-1}\right)} w_{l}\left(\frac{2 l x}{L}\right) .
$$


By scaling

$$
y=\frac{2 l}{L} x, \quad \Phi(y)=\phi(x)
$$

it is easy to see that (5.1) is equivalent to the following

$$
\left\{\begin{array}{l}
\Phi_{y y}-\Phi+3 w_{l}^{2} \Phi-\gamma\left(\int_{0}^{l} w_{l} \Phi d y\right) w_{l}=\lambda \Phi, \quad 0<y<l \\
\Phi_{y}(0)=\Phi_{y}(l)=0
\end{array}\right.
$$

where

$$
\gamma=\frac{2 \mu^{\prime}}{l\left(\mu^{\prime}-1\right)}=\frac{2 t}{l}>0
$$

and $w_{l}$ is the unique solution of (2.7). (Note that (5.3) is self-adjoint.)

In this section, we give a complete study of (5.3).

We first have

Lemma 10. $\lambda=0$ is an eigenvalue of (5.3) if and only if

$$
\gamma \int_{0}^{l} w_{l} \mathcal{L}^{-1} w_{l} d y=1
$$

Proof: Suppose $\lambda=0$. Then we have

$$
0=\mathcal{L}[\Phi]-\gamma\left(\int_{0}^{l} w_{l} \Phi d y\right) w_{l}
$$

which implies that

$$
\Phi=\gamma\left(\int_{0}^{l} w_{l} \Phi d y\right) \mathcal{L}^{-1} w_{l}
$$

Multiplying (5.6) by $w_{l}$ and integrating, we obtain (5.5) since $\int_{0}^{l} w_{l} \Phi d y \neq 0$ (as otherwise $\mathcal{L} \Phi=0$ and hence $\Phi=0)$.

The following is the main result of this section:

Lemma 11. All eigenvalues of (5.3) are real and

(a) if $\gamma \int_{0}^{l} w_{l} \mathcal{L}^{-1} w_{l} d y>1$, then for all eigenvalues of (5.3) we have $\lambda<0$;

(b) if $\gamma \int_{0}^{l} w_{l} \mathcal{L}^{-1} w_{l} d y=1$, then for all eigenvalues of (5.3) we have $\lambda \leq 0$ and zero is an eigenvalue of (5.3) with eigenfunction $\mathcal{L}^{-1} w_{l}$;

(c) if $\gamma \int_{0}^{l} w_{l} \mathcal{L}^{-1} w_{l} d y<1$, then there exists an eigenvalue $\lambda_{0}>0$ of (5.3).

From Lemma 11, we see that $\gamma \int_{0}^{l} w_{l} \mathcal{L}^{-1} w_{l} d y=1$ is the borderline case between stability and instability of (5.3).

Proof: The nonlocal eigenvalue problem (5.3) is self-adjoint and hence all eigenvalues are real. Let $\lambda \geq 0$ be an eigenvalue of (5.3). We first claim that $\lambda \neq \lambda_{1}$, where $\lambda_{1}$ is the first eigenvalue of $\mathcal{L}$ given by Lemma 8 . In fact, if $\lambda=\lambda_{1}$, then we have

$$
\gamma \int_{0}^{l} w_{l} \Phi_{1} d y=0
$$


where $\Phi_{1}$ is the eigenfunction to the eigenvalue $\lambda_{1}$ for the operator $\mathcal{L}$. This implies

$$
\int_{0}^{l} w_{l} \Phi_{1} d y=0
$$

which is impossible since $\Phi_{1}>0$.

So $\lambda \neq \lambda_{1}$. By Lemma 8, $(\mathcal{L}-\lambda)^{-1}$ exists and hence $\lambda>0$ is an eigenvalue of (5.3) if and only if it satisfies the following algebraic equation:

$$
1-\gamma \int_{0}^{l}\left[\left((\mathcal{L}-\lambda)^{-1} w_{l}\right) w_{l}\right] d y=0 .
$$

Let

$$
\rho(t)=1-\gamma \int_{0}^{l}\left[\left((\mathcal{L}-\lambda)^{-1} w_{l}\right) w_{l}\right] d y, \quad t \geq 0, \quad t \neq \lambda_{1} .
$$

Then $\rho(0)=1-\gamma \int_{0}^{l}\left(w_{l} \mathcal{L}^{-1} w_{l}\right) d y$ and

$$
\rho^{\prime}(t)=-\gamma \int_{0}^{L}\left[\left((\mathcal{L}-t)^{-2} w_{l}\right) w_{l}\right] d y<0 .
$$

On the other hand,

$$
\begin{gathered}
\rho(t) \rightarrow-\infty \text { as } t \rightarrow \lambda_{1}, t<\lambda_{1} \\
\rho(t) \rightarrow+\infty \text { as } t \rightarrow \lambda_{1}, t>\lambda_{1} \\
\rho(t) \rightarrow 1 \text { as } t \rightarrow+\infty .
\end{gathered}
$$

Thus $\rho(t)>0$ for $t>\lambda_{1}$ and $\rho(t)$ has a (unique) zero in $\left(0, \lambda_{1}\right)$ if and only if $\rho(0)>0$ which is equivalent to $1-\gamma \int_{0}^{l}\left(w_{l} \mathcal{L}^{-1} w_{l}\right) d y>0$.

This proves the lemma.

\section{The Proof of Theorem 3}

Now we can finish the proof of Theorem 3.

By Lemma 11, we have stability of (5.3) if

$$
\gamma \int_{0}^{l}\left(w_{l} \mathcal{L}^{-1} w_{l}\right) d y>1
$$

and instability if

$$
\gamma \int_{0}^{l}\left(w_{l} \mathcal{L}^{-1} w_{l}\right) d y<1
$$

By Lemma 9, we have

$$
\gamma \int_{0}^{l}\left(w_{l} \mathcal{L}^{-1} w_{l}\right) d y=\frac{t \frac{d}{d l}\left(l \int_{0}^{l} w_{l}^{2} d y\right)}{2 l}
$$

Thus (6.1) is equivalent to

$$
2 l-t \frac{d}{d l}\left(l \int_{0}^{l} w_{l}^{2} d y\right)<0
$$

which, by definition (3.29), is equivalent to

$$
\frac{d \tilde{f}}{d l}<0
$$


Thus, for $\mu_{2}<\mu^{\prime}<\mu_{1}$, by Lemma 7 (c), the solution with small period $l_{1}$ is stable while the solution with large period $l_{2}$ is unstable. For $\mu^{\prime} \leq \mu_{2}$, the only solution is unstable. When $\mu^{\prime}=\mu_{1}$, we have $\tilde{f}^{\prime}(l)=0$ which implies that the only solution is neutrally stable.

When $\mu_{2}<\mu^{\prime}<\mu_{1}$, let us compute the amplitude of $A$ :

$$
A(x)=\frac{2 l}{L \sqrt{\mu^{\prime}-1}} w_{l}\left(\frac{2 l x}{L}\right) .
$$

So the maximum of $A(x)$ is given by

$$
\begin{gathered}
A(0)=\max _{x \in I} A(x)=\frac{2 l}{L \sqrt{\mu^{\prime}-1}} w_{l}(0) \\
=\frac{2 l}{L \sqrt{\mu^{\prime}-1}} M \\
=\frac{2}{L \sqrt{\mu^{\prime}-1}} \sqrt{2-k^{2}} K(k) \frac{1}{\sqrt{1-\frac{k^{2}}{2}}} \\
=\frac{2 \sqrt{2}}{L \sqrt{\mu^{\prime}-1}} K(k) .
\end{gathered}
$$

So if $l_{1}<l_{2}$, then by (3.12) $k_{1}<k_{2}$ and the maximum of $A$ for $l_{1}$ is smaller than the maximum of $A$ for $l_{2}$.

Thus, for $\mu_{2}<\mu^{\prime}<\mu_{1}$, the solution with small amplitude is stable and the one with large amplitude is unstable.

This finishes the proof of Theorem 3.

\section{Instability of other Solutions: The PRoof of theorem 2}

In this section we will show that all other solutions with minimal period $2 l$ must be unstable. In fact, let $w$ be any solution of the following ordinary equation:

$$
w^{\prime \prime}-w+w^{3}=0, \quad w^{\prime}(0)=w^{\prime}(l)=0
$$

and consider its associated eigenvalue problem

$$
\left\{\begin{array}{l}
\mathcal{L} \phi=\phi^{\prime \prime}-\phi+3 w^{2} \phi=\lambda \phi, \quad 0<y<l \\
\phi^{\prime}(0)=\phi^{\prime}(l)=0
\end{array}\right.
$$

The Morse index of the a solution $w$ of (7.1) is the number of positive eigenvalues of (7.2).

Since $w_{l}$ is the unique positive solution of (7.1) it has least energy under all solutions of (7.1) and hence its Morse index is 1.

We claim that

Lemma 12. All other solutions of (7.1) have Morse index at least 2.

Proof: Let $w \neq w_{l}$ be a solution of (7.1). Let $\lambda_{1}>0$ be the principal eigenvalue of $w$. The associated eigenfunction $\Phi_{1}$ can be made positive. By definition,

$$
-\lambda_{2}=\inf _{\phi \in H^{1}(0, l), \phi \neq 0, \int_{0}^{l} \Phi_{1} \phi d y=0} \frac{\int_{0}^{l}\left[\left(\phi^{\prime}\right)^{2}+\phi^{2}-3 w^{2} \phi^{2}\right] d y}{\int_{0}^{l} \phi^{2} d y} .
$$


We now show that $\lambda_{2}>0$. There are two cases to be considered.

Case 1. $w$ is a changing-sign solution. In this case, we suppose that $w(x)>0, x \in\left(0, x_{1}\right)$ and $w(x)<0, x \in\left(x_{1}, x_{2}\right)$ where $x_{2} \leq l$. We may assume that $w\left(x_{1}\right)=0$ and $w\left(x_{2}\right)=0$ if $x_{2}<l$ and $w^{\prime}\left(x_{2}\right)=0$ if $x_{2}=l$. Now let $\phi(x)=c_{1} w(x), x \in\left(0, x_{1}\right)$ and $\phi(x)=c_{2} w(x), x \in\left(x_{1}, x_{2}\right)$. We can choose the two constants $c_{1}, c_{2}$ such that

$$
\int_{0}^{l} \Phi_{1} \phi d y=0
$$

Then, by simple computations, we have

$$
\int_{0}^{l}\left[\left(\phi^{\prime}\right)^{2}+\phi^{2}-3 w^{2} \phi^{2}\right] d y<0
$$

which implies that $-\lambda_{2}<0$ and hence $\lambda_{2}>0$.

Case 2. $w$ is a positive solution. Since $w \neq w_{l}, w^{\prime}$ must change sign. We may assume that $w^{\prime}<0, x \in$ $\left(0, x_{1}\right)$ and $w^{\prime}>0, x \in\left(x_{1}, x_{2}\right)$. Furthermore, we may also assume that $w^{\prime}\left(x_{1}\right)=0$ and $w^{\prime}\left(x_{2}\right)=0$. Then similar to Case 1, we take $\phi=c_{1} w^{\prime}(x), x \in\left(0, x_{1}\right)$ and $\phi(x)=c_{2} w^{\prime}(x), x \in\left(x_{1}, x_{2}\right)$, where the constants $c_{1}, c_{2}$ are chosen such that $\int_{0}^{l} \Phi_{1} \phi d y=0$. Then, by simple computations, we have

$$
\int_{0}^{l}\left(\left(\phi^{\prime}\right)^{2}+\phi^{2}-3 w^{2} \phi^{2}\right) d y \leq 0
$$

and hence $-\lambda_{2} \leq 0$ and $\lambda_{2} \geq 0$. If $\lambda_{2}=0$, then $\phi(x)$ becomes an eigenfunction which satisfies (7.2) and hence is smooth. This is impossible since $\phi$ is not smooth at $x_{1}$ (as otherwise $w^{\prime}\left(x_{1}\right)=w^{\prime \prime}\left(x_{1}\right)=0$ and hence $w \equiv 1)$.

From Lemma 12 it follows that the Morse index of all other solutions is at least 2 . In other words, problem (7.2) has at least two positive eigenvalues. Let $\lambda_{1}$ be the principal eigenvalue and $0<\lambda_{2} \leq \lambda_{1}$ be the second eigenvalue. Let the corresponding eigenfunctions be $\Phi_{1}, \Phi_{2}$. Since $\lambda_{1}$ is the principal eigenvalue, we may assume that $\Phi_{1}>0$.

If $\int_{0}^{l} w \Phi_{2} d y=0$, we choose $\phi=\Phi_{2}$. If $\int_{0}^{l} w \Phi_{2} d y \neq 0$, then we choose $c$ such that

$$
\int_{0}^{l} w \Phi_{1} d y+c \int_{0}^{l} w \Phi_{2} d y=0
$$

and

$$
\phi=\Phi_{1}+c \Phi_{2}
$$

In any case, we obtain that $\int_{0}^{l} w \phi d y=0$.

Then we have

$$
\begin{gathered}
\int_{0}^{l}\left[\left(\phi^{\prime}\right)^{2}+\phi^{2}-3 w^{2} \phi^{2}\right] d y-\gamma\left(\int_{0}^{l} w_{l} \phi d y\right)^{2} \\
=\int_{0}^{l}\left(\left(\phi^{\prime}\right)^{2}+\phi^{2}-3 w_{l}^{2} \phi^{2}\right) d y<0
\end{gathered}
$$

which implies that for any constant $\gamma$ there exists a positive eigenvalue to (5.3) and, by the scaling argument in Section 5, also to (5.1). Now, by the reduction lemma (Lemma 13), all periodic solutions of (1.5) which do not have minimal period $L$ must be unstable.

This finishes the proof of Theorem 2. 


\section{Appendix: The Linearized Operator - A Reduction Lemma}

In this appendix, we derive the linearized operator to system (1.5) and study some of its properties.

In particular, we show that its eigenvalues must all be real and that the system of eigenvalue equations reduces to a self-adjoint nonlocal eigenvalue problem. This is similar to our previous paper [8]. For the sake of completeness, we include all this material in this Appendix.

To study the linear stability of $(1.5)$, we perturb $(A(x), B(x))$ as follows:

$$
A_{\epsilon}(x, t)=A(x)+\epsilon \phi(x) e^{\lambda_{L} t}, \quad B_{\epsilon}(x, t)=B(x)+\epsilon \psi(x) e^{\lambda_{L} t},
$$

where $\lambda_{L} \in \mathcal{C}$ - the set of complex numbers - and

$$
\phi, \psi \in \mathcal{X}_{L}
$$

where $\mathcal{X}_{L}$ was defined at the beginning of Section 5 .

Substituting (7.5) into (1.2) and considering the linear part, we obtain the following eigenvalue problem:

$$
\left\{\begin{array}{l}
\phi_{x x}+(1-B) \phi-3 A^{2} \phi-A \psi=\lambda_{L} \phi, \quad-\frac{L}{2}<x<\frac{L}{2}, \\
\psi_{x x}+2 \mu^{\prime}(A \phi)_{x x}=\tau \lambda_{L} \psi, \quad-\frac{L}{2}<x<\frac{L}{2} \\
\lambda_{L} \in \mathcal{C}, \phi, \psi \in \mathcal{X}_{L} .
\end{array}\right.
$$

Let

$$
\psi=-2 \mu^{\prime} A \phi+2 \mu^{\prime}<A \phi>+\tau \lambda_{L} \hat{\psi}
$$

where

$$
<\hat{\psi}>=0
$$

Equation (7.7) together with (7.6) implies

$$
\hat{\psi}_{x x}-\tau \lambda_{L} \hat{\psi}=-2 \mu^{\prime} A \phi+2 \mu^{\prime}<A \phi>.
$$

Substituting (2.1) and (7.7) into the first equation of (7.6), we obtain that

$$
\phi_{x x}-a \phi+3 b A^{2} \phi-2 \mu^{\prime}<A \phi>A-\tau \lambda_{L} A \hat{\psi}=\lambda_{L} \phi,-\frac{L}{2}<x<\frac{L}{2},
$$

where $a$ and $b$ are given by (2.3).

If $\tau=0$, then (7.9) becomes

$$
\left\{\begin{array}{l}
\phi_{x x}-a \phi+3 b A^{2} \phi-2 \mu^{\prime}<A \phi>A=\lambda_{L} \phi, \quad-\frac{L}{2}<x<\frac{L}{2}, \\
\phi \in \mathcal{X}_{L}
\end{array}\right.
$$

We now recall the following reduction lemma (Lemma 3 of [8]):

Lemma 13. (a) All eigenvalues of (7.6) are real.

(b) If all eigenvalues of (7.10) are negative, then all eigenvalues of (7.6) are negative.

(c) If problem (7.10) has a positive eigenvalue, then problem (7.6) also has a positive eigenvalue.

Lemma 13 implies that the stability and instability properties of (7.6) and (7.10) are the same. Thus we have reduced our stability problem to the study of the self-adjoint nonlocal eigenvalue problem (7.10). 
We first prove part (a). Multiplying (7.9) by $\bar{\phi}-$ the conjugate function of $\phi$ - and integrating over $I$, we obtain

$$
\begin{gathered}
\lambda_{L} \int_{I}|\phi|^{2} d x=-\int_{I}\left[\left|\phi_{x}\right|^{2}+a|\phi|^{2}-3 b A^{2}|\phi|^{2}\right] d x-\frac{2 \mu^{\prime}}{L}\left|\int_{I}(A \phi) d x\right|^{2} \\
-\tau \lambda_{L} \int_{I} A \hat{\psi} \bar{\phi} d x .
\end{gathered}
$$

Multiplying the conjugate of (7.8) by $\hat{\psi}$ and integrating over $R$ we get

$$
\int_{I} A \bar{\phi} \hat{\psi} d x=\frac{1}{2 \mu^{\prime}} \int_{I}\left|\hat{\psi}_{x}\right|^{2} d x+\frac{\tau \bar{\lambda}_{L}}{2 \mu^{\prime}} \int_{I}|\hat{\psi}|^{2} d x
$$

Substituting (7.12) into (7.11) gives

$$
\begin{aligned}
\lambda_{L} \int_{I}|\phi|^{2} d & +\int_{I}\left[\left|\phi_{x}\right|^{2}+a|\phi|^{2}-3 b A^{2}|\phi|^{2}\right] d x+\frac{2 \mu^{\prime}}{L}\left|\int_{I}(A \phi) d x\right|^{2} \\
+ & \frac{\tau \lambda_{L}}{2 \mu^{\prime}} \int_{I}\left|\hat{\psi}_{x}\right|^{2} d x+\frac{\tau^{2}\left|\lambda_{L}\right|^{2}}{2 \mu^{\prime}} \int_{I}\left|\hat{\psi}_{x}\right|^{2} d x=0 .
\end{aligned}
$$

Taking the imaginary part of (7.13), we obtain

$$
\lambda_{i}\left(\int_{I}|\phi|^{2} d x+\frac{\tau}{2 \mu^{\prime}} \int_{I}\left|\hat{\psi}_{x}\right|^{2} d x\right)=0
$$

where $\lambda_{L}=\lambda_{r}+\sqrt{-1} \lambda_{i}$.

Equation (7.14) implies

$$
\lambda_{i}=0
$$

and therefore $\lambda_{L}$ is real. Since the spectrum of (7.9) coincides with that of (7.6) the proof of part (a) is complete.

Next we prove parts (b) and (c) of Lemma 13. We use variational techniques. To this end, we need to introduce two quadratic forms: Let

$$
L[\phi]=\int_{I}\left(\left|\phi_{x}\right|^{2}+a|\phi|^{2}-3 b A^{2}|\phi|^{2}\right) d x+\frac{2 \mu^{\prime}}{L}\left|\int_{I} A \phi d x\right|^{2}, \quad \phi \in \mathcal{X}_{L}
$$

and

$$
\mathcal{L}_{\lambda}[\phi]=L[\phi]+\frac{\tau \lambda}{2 \mu^{\prime}} \int_{I}\left(\left|\hat{\psi}_{x}\right|^{2}+\tau \lambda|\hat{\psi}|^{2}\right) d x
$$

where $\hat{\psi}$ is the unique solution of the problem

$$
\left\{\begin{array}{l}
\hat{\psi}^{\prime \prime}-\tau \lambda \hat{\psi}=-2 \mu^{\prime} A \phi+2 \mu^{\prime}<A \phi>, \\
\hat{\psi} \in \mathcal{X}_{L}, \quad<\hat{\psi}>=0 .
\end{array}\right.
$$

Observe that for $\tau \geq 0$ and $\lambda \geq 0$

$$
\mathcal{L}_{0}[\phi]=L[\phi], \quad L[\phi] \leq \mathcal{L}_{\lambda}[\phi] .
$$

To prove (b), we note that if all eigenvalues of (7.10) are neative, then the quadratic form $L[\phi]$ is positive definite, which by (7.19) yields that $\mathcal{L}_{\lambda}$ is positive definite if $\lambda \geq 0$. Let $\lambda \geq 0$ be an eigenvalue of (7.6), then, by (7.13), we obtain that

$$
\lambda \int_{I}|\phi|^{2} d x+\mathcal{L}_{\lambda}[\phi]=0
$$


which is clearly impossible if $\lambda \geq 0$. Thus we have shown that all eigenvalues of (7.6) must be negative.

To prove (c), suppose that (7.10) has a positive eigenvalue. Then the eigenvalue problem

$$
-\mu_{L}=\min _{\phi \in \mathcal{X}_{L}, \int_{I} \phi^{2} d x=1} L[\phi]
$$

has a positive value $\mu_{L}>0$. We now claim that (7.6) admits a positive eigenvalue.

Fixing $\lambda \in[0,+\infty)$, let us consider another eigenvalue problem

$$
-\mu(\lambda)=\min _{\phi \in \mathcal{X}_{L}, \int_{I} \phi^{2} d x=1} \mathcal{L}_{\lambda}[\phi]
$$

A minimizer $\phi$ of (7.22) satisfies the equation

$$
\phi_{x x}-a \phi+3 b A^{2} \phi-2 \mu^{\prime}<A \phi>A-A \hat{\psi}=\mu(\lambda) \phi, \quad \phi \in \mathcal{X}_{L}
$$

where $\hat{\psi}$ is given by $(7.18)$.

By $(7.19),-\mu(\lambda) \geq-\mu_{L}$. Hence $\mu(\lambda) \leq \mu_{L}$. Moreover, since $\hat{\psi}$ is continuous with respect to $\lambda$ in $[0,+\infty)$, we see that $\mu(\lambda)$ is also continuous in $[0,+\infty)$.

Let us consider the following algebraic equation

$$
h(\lambda):=\mu(\lambda)-\lambda=0, \quad \lambda \in[0,+\infty) .
$$

By our assumption, $h(0)=\mu(0)=\mu_{L}>0$. On the other hand, for $\lambda>2 \mu_{L}, h(\lambda) \leq \mu_{L}-\lambda<-\mu_{L}<0$. By the mean-value theorem, there exists a $\lambda_{L} \in\left(0, \mu_{L}\right)$ such that $h\left(\lambda_{L}\right)=0$.

Substituting $\mu\left(\lambda_{L}\right)=\lambda_{L}$ into (7.23), we see that $\lambda_{L}$ is an eigenvalue of problem (7.6).

Part (c) of Lemma 13 is thus proved.

\section{REFERENCES}

[1] M. Abramowitz and I.A. Stegun, Handbook of Mathematical Functions with Formulas, Graphs, and Mathematical Tables, Washington, National Bureau of Standards Applied Mathematics, 1964.

[2] P.F. Byrd and M.D. Friedman, Handbook of elliptic integrals for engineers and scientists, Springer-Verlag, 1971.

[3] P. Coullet and G. Iooss, Instabilities of one-dimensional cellular patterns, Phys. Rev. Lett. 64 (1990), 866-869.

[4] S.M. Cox and P.C. Matthews, New instabilities in two-dimensional rotating convection and magnetoconvection, Phys. D 149 (2001), 210-229.

[5] S. Fauve, Pattern forming instabilities, pp. 387-491, in "Hydrodynamics and nonlinear instabilities", C. Godrèche and P. Manneville ed., Cambridge University Press, 1998.

[6] C.-S. Lin and W.-M. Ni, On the diffusion coefficient of a semilinear Neumann problem, Calculus of variations and partial differential equations (Trento, 1986) 160-174, Lecture Notes in Math., 1340, Springer, Berlin-New York, 1988.

[7] P.C. Matthews and S.M. Cox, Pattern formation with a conservation law, Nonlinearity 13 (2000), 1293-1320.

[8] J. Norbury, M. Winter and J. Wei, Existence and stability of singular patterns in a Ginzburg-Landau equation coupled with a mean field, Nonlinearity 15 (2002), 2077-2096.

[9] H. Riecke, Localized structures in pattern-forming systems, in "Pattern formation in continuous and coupled systems " (Minneapolis, MN, 1998), 215-229, IMA Vol. Math. Appl. 115

[10] J. Wei, On single interior spike solutions of the Gierer-Meinhardt system: uniqueness and spectrum estimates, Euro. J. Appl. Math. 10 (1999), 353-378. 
Mathematical Institute, University of Oxford, 24-29 St. Giles', Oxford, UK

E-mail address: john.norbury@lincoln.oxford.ac.uk

Department of Mathematics, The Chinese University of Hong Kong, Shatin, Hong Kong

E-mail address: wei@math.cuhk.edu.hk

Department of Mathematical Sciences, Brunel University, Uxbridge UB8 3PH, United Kingdom

E-mail address: matthias.winter@brunel.ac.uk 